\title{
Sífilis congênita no recém-nascido: repercussões para a mãe
}

\author{
Congenital syphilis in newborns: repercussions for the mother \\ Sifilis congénita en el neonato: repercusiones para la madre
}

\author{
Jéssica Gama da Silva'; Giovana Calcagno Gomes"; Juliane Portella Ribeiro"l'; \\ Camila Magroski Goulart Nobre ${ }^{\text {iv }}$; Pâmela Kath de Oliveira Nörberg ${ }^{v}$; Marina Soares Mota ${ }^{\text {I }}$
}

\begin{abstract}
RESUMO
Objetivo: conhecer as repercussões do diagnóstico da Sífilis Congênita no recém-nascido para a mãe. Método: pesquisa qualitativa desenvolvida com 15 mães de recém-nascidos com Sífilis Congênita e internados em um hospital público do sul do Brasil. Os dados foram coletados por entrevistas semiestruturadas, submetidos à análise de conteúdo e aprovados pelo Comitê de Ética em Pesquisa. Resultados: as mães manifestaram sentimentos de culpa, desespero, tristeza e horror. Havia esperança de não passar a infeç̧ão para o recém-nascido. Verificou-se a reincidência da doença em mais de uma gestação. Referiram medo do estigma social e buscaram informações acerca da doença na internet, como também com médicos e enfermeiros. Conclusão: há desinformação das mães quanto à infecção da sífilis, principalmente sobre como evitar a transmissão vertical e a reinfecção. Cabe ao enfermeiro, instrumentalizar a mãe para o cuidado ao recém-nascido com Sífilis Congênita, por meio de um processo educativo que qualifique o cuidado da criança e da mãe.
\end{abstract}

Descritores: Sífilis congênita; transmissão vertical de doença infecciosa; família; enfermagem.

\section{ABSTRACT}

Objective: to learn how a diagnosis of congenital syphilis in newborns affects the mothers. Method: in this qualitative study of 15 mothers of newborns with congenital syphilis admitted to a public hospital in southern Brazil, data were collected by semistructured interview, and treated by content analysis. The study was approved by the research ethics committee. Results: the mothers expressed feelings of guilt, despair, sadness and horror. They hoped not to transmit the infection to the newborn. The disease was found to have recurred in more than one pregnancy. They reported fear of social stigma, and looked for information about the disease on the Internet, as well as from doctors and nurses. Conclusion: mothers are misinformed regarding infection by syphilis, especially on how to prevent vertical transmission and reinfection. It is up to nurses to instruct mothers on how to care for newborns with congenital syphilis, through an educational process to improve care for the child and the mother. Descriptors: Syphilis, congenital; infectious disease transmission, vertical; family; nursing.

\section{RESUMEN}

Objetivo: conocer las repercusiones, para la madre, del diagnóstico de sífilis congénita en el neonato. Método: investigación cualitativa desarrollada junto a 15 madres de neonatos con sífilis congénita e ingresados en un hospital público en el sur de Brasil. Se recolectaron los datos a través de entrevistas semiestructuradas y se sometieron al análisis de contenido; después fueron aprobados por el Comité de Ética en Investigación. Resultados: las madres expresaron sentimientos de culpa, desesperación, tristeza y horror. Había esperanza de no transmitir la infección al neonato. Se verificó la reincidencia de la enfermedad en más de un embarazo. Declararon el temor al estigma social y buscaron información sobre la enfermedad en internet, así como junto a médicos y enfermeros. Conclusión: las madres están desinformadas en cuanto a la infección por Sífilis, especialmente sobre cómo prevenir la transmisión vertical y la reinfección. Le toca al enfermero darle a la madre herramientas para que cuide bien al neonato con Sífilis Congénita, a través de un proceso educativo que califique el cuidado del niño y la madre.

Descriptores: Sífilis congénita; transmisión vertical de enfermedad infecciosa; familia; enfermería.

\section{INTRODUÇÃO}

A infecção da criança pelo Treponema pallidum, a partir da placenta da mãe acarreta o desenvolvimento da Sífilis Congênita (SC). A transmissão pelo contato do recém-nascido com lesões genitais no momento do parto também pode acontecer $^{1}$. A sífilis na gravidez causa aproximadamente 300.000 mortes fetais e neonatais/ano e coloca 215.000 recémnascidos (RN) sob o risco de morte prematura, baixo peso ao nascimento ou $\mathrm{SC}^{1,2}$.

'Enfermeira. Mestre em Enfermagem. Universidade Federal do Rio Grande. Brasil. E-mail: jehgama92@gmail.com

"Enfermeira. Doutora em Enfermagem, Docente Titular. Universidade Federal do Rio Grande. Brasil. E-mail: giovanacalcagno@furg.br

I'Enfermeira. Doutora em Enfermagem. Docente Adjunta. Universidade Federal de Pelotas. Brasil. E-mail: ju_ribeiro1985@hotmail.com

IVEnfermeira. Doutoranda em Enfermagem. Universidade Federal do Rio Grande. Brasil. E-mail: kamy_magroski@yahoo.com.br

vEnfermeira. Doutora em Enfermagem, Universidade Federal do Rio Grande. Rio Grande do Sul. Brasil. E-mail: pamelakathpko@yahoo.com.br

V'Enfermeira. Doutora em Enfermagem. Docente Adjunta. Universidade Federal do Rio Grande. Brasil. E-mail: msm.mari.gro@gmail.com 
Ao receber o diagnóstico de SC do filho muitas mães ficam surpresas, pois não esperam que o RN também possa contrair a infecção ${ }^{3}$. Nesse momento, torna-se importante o papel do enfermeiro no apoio e orientação à mãe, minimizando dúvidas sobre o diagnóstico, tratamento e os cuidados tanto com ela quanto com o RN. A questão que norteou esse estudo foi: Qual a repercussão para a mãe, frente ao diagnóstico de SC no RN? A partir desse questionamento, objetivou-se conhecer as repercussões do diagnóstico da SC no RN para a mãe. O conhecimento gerado neste estudo poderá subsidiar os profissionais da saúde/enfermeiros na instrumentalização da família no cuidado à criança com SC.

\section{REVISÃO DE LITERATURA}

Muitas mães ao serem informadas sobre o diagnóstico da SC em seu filho apresentam tristeza e culpa por ter-lhe transmitido a infecção, temendo as complicações da doença ${ }^{3}$. Apesar do diagnóstico de sífilis na mãe ser confirmado durante o pré-natal muitas mulheres não realizam o tratamento ou se reinfectam. Estudo mostra a má qualidade do atendimento do pré-natal como um prejuízo à gestante, pois esse é um momento para a detecção precoce de várias doenças e seu tratamento imediato ${ }^{4}$.

Algumas mães só percebem a repercussão da SC quando seus filhos têm que permanecer internados para realização do tratamento. A pouca compreensão acerca da doença somada à baixa escolarização contribuem para que resistam à hospitalização do RN após o parto ${ }^{5}$.

O diagnóstico de SC no RN pode gerar conflitos nos relacionamentos conjugais ${ }^{6}$. Estudo apontou o não tratamento adequado das mães e seus parceiros durante a gestação ${ }^{7}$. Faz-se necessário que as mães recebam orientações acerca dos procedimentos a serem realizados no RN, bem como a realização dos exames periódicos e a evolução clínica esperada. Os profissionais devem apresentar-se disponíveis, esclarecendo suas dúvidas, de modo que o tempo vivido no hospital seja significativo, sendo-Ihe fonte de apoio ${ }^{8}$.

O enfermeiro deve compreender as repercussões da hospitalização da criança, pois ocorre mudança de rotina, ausência da mãe no lar, conflitos conjugais e tensão em relação ao filho doente ${ }^{9}$. A terapêutica da SC resulta na administração endovenosa de antibiótico o que exige várias punções. Além disso, o tratamento é doloroso e contempla, também, a realização de exames de sangue, Raios $X$ dos ossos longos e punção lombar, entre outros ${ }^{10}$.

\section{METOdOLOGIA}

Estudo descritivo com abordagem qualitativa. Participaram 15 mães de RN internados com diagnóstico de SC. O critério de inclusão consistiu em ser mãe acompanhante da criança durante seu período de internação. Foram excluídas mães menores de 18 anos. As mães foram selecionadas aleatoriamente e convidadas pessoalmente para participação do estudo pela pesquisadora, sendo explicados o objetivo, a metodologia e o método de coleta dos dados.

Os dados foram coletados entre os meses de maio e julho de 2018, em uma Unidade de Pediatria de um hospital no sul do Brasil. Os dados foram coletados através de entrevistas semiestruturadas. Ao aceitarem, assinaram o Termo de Consentimento Livre e Esclarecido. A entrevista foi realizada em sala anexa à unidade. Foram questionadas acerca do impacto do diagnóstico da SC para elas. As entrevistas foram gravadas e transcritas.

Aplicou-se a técnica análise de conteúdo do depoimentos operacionalizada em três etapas: Pré-análise na qual se realizou a leitura flutuante dos dados e formulação de hipóteses; Exploração do material, em que ocorreu a codificação dos dados e sua classificação desses em categorias; e Tratamento dos resultados obtidos, Inferência, Interpretação e Reflexão, ocasião em que os dados foram discutidos ${ }^{11}$. Emergiram como categorias: Sentimentos frente ao diagnóstico e ao tratamento da SC do RN; A reincidência da SC em mais de uma gestação; O medo do estigma que envolve a doença; e a busca de informações acerca da SC.

Foram respeitados os princípios éticos da pesquisa envolvendo seres humanos, conforme a Resolução no 466/12. Todos os participantes foram identificados com a letra F, para garantir o anonimato, seguida do número arábico correspondente à ordem de participação nas entrevistas. O projeto de pesquisa foi aprovado pelo Comitê de Ética com o parecer no 33/2018.

\section{RESULTADOS E DISCUSSÃO}

Participaram 15 mães, com idades entre 18 e 46 anos. Duas tinham o Ensino Superior completo, uma Ensino Superior incompleto, quatro o Ensino Médio, cinco o Ensino Médio incompleto, uma possuía o Ensino Fundamental completo e duas o Ensino Fundamental incompleto. Duas eram assistentes administrativas, uma era enfermeira, três estudantes e nove do lar. Dez tinham relacionamentos conjugais estáveis e cinco eram solteiras. Suas rendas familiares variaram entre um e cinco salários mínimos regionais brasileiros.

As categorias emergentes dos depoimentos são tratadas a seguir. 


\section{Sentimentos frente ao diagnóstico e ao tratamento da SC do RN}

As mães receberam o diagnóstico de SC do RN por meio de profissionais da saúde. Frente ao diagnóstico manifestaram culpa, desespero, tristeza e horror. Esses se intensificaram ao acompanhar o processo doloroso do tratamento na criança.

Eu não sabia, fiquei sabendo depois que fizeram o exame nele de sangue. Eu não sabia que ele ia ter que ficar aqui 10 dias, porque ele estava com sifilis. É sofrido ver furar ele toda hora. [...]. (F2)

Eu fiquei muito arrasada [...] a gente achava que ia embora com ele e, no fim, tivemos que ficar. (F10)

A pediatra me falou. Senti que eu tinha sido irresponsável, sabe? [...] Eu me senti culpada. (F9)

[...] me senti sem chão, desesperada. O pediatra que me passou o diagnóstico dele. Disse que estavam suspeitando de SC. (F12)

As participantes ressaltaram que tinham esperanças que, após o tratamento, não passariam para os RN a infecção da sífilis. Tinham receio sobre os sinais e sintomas que eles poderiam vir a ter.

Fiz o tratamento, mas tinha medo do que poderia vir a acontecer com a minha filha, [...] como ela ia ser gerada, que doenças isso poderia acarretar? [...] era muita preocupação junta. (F14)

Foi horrivel, pois eu fiz o tratamento achando que ela não ia precisar fazer. [...] ela nasceu, fizeram os exames e deu que ela tinha também. Chorei muito, todo dia que dão remédio, ela chora e eu choro junto! Porque é bem dolorido! (F7)

Apenas uma mãe relatou que sabia da possibilidade de passar a doença para seu filho desde o início do tratamento e destacou que a orientação recebida pelos profissionais de saúde como importante auxílio para aceitação do diagnóstico na criança. Outras mães só souberam o diagnóstico após o nascimento, mas o fato de ter tratamento para a infecção às deixou mais tranquilas para o seu enfrentamento.

Eu já sabia que ele poderia ter. Quando ela falou (médica) o susto até que não foi tanto [...]. Como eu só comecei o tratamento no segundo trimestre, ela disse que o risco de passar para ele era maior. [...] (F5)

Eu não sabia. Fiquei sabendo depois que fizeram o exame nele de sangue. Reagi mal, mas sabia que tinha tratamento. [...]. (F2)

Eu pensei que ela não tinha, porque pelo tratamento, o médico da Unidade falou que ela poderia não ter a doença. Eu descobri [...] só depois que ela nasceu [...]. (F6)

Frente ao exposto, verificou-se que tanto o diagnóstico quanto o tratamento da SC no RN geram sentimentos negativos na mãe. Estudo também apontou preocupação, tristeza e culpa pela contaminação do filho ${ }^{3}$. Outro apresentou que os pais possuem sentimentos de tristeza, pavor, preocupação e medo do filho nascer com problemas de saúde decorrentes da $\mathrm{SC}^{12}$. A necessidade da hospitalização pode gerar sentimentos como sofrimento, impotência, estresse, preocupação e ansiedade relacionada às múltiplas punções venosas, ao risco de morte iminente e a espera dos resultados dos exames laboratoriais e de imagem ${ }^{12}$.

Estudo apontou que os RN infectados indicam gestantes que não realizaram tratamento 19 (35,2\%) ou com diagnóstico tardio 50 (92,6\%). Entre os RN não infectados quatro (19,0\%) não realizaram tratamento algum e 14 (77,8\%) apresentaram diagnóstico tardio ${ }^{13}$. Segundo análise dos casos de SC, entre os anos de 2007 a 2014 em Palmas, observou-se que 161 (78,9\%) dos RN foram classificados como vivos; cinco (2,5\%) tiveram óbito por sífilis; sete (3,4\%) foram a óbito por outras causas; $19(9,3 \%)$ foram aborto e 12 (5,9\%) natimortos ${ }^{7}$. Dados nacionais revelaram que de 24.098 nascidos com SC 2.268 (9,5\%) foram RN com baixo peso, 2.854 (11,8\%) prematuros, 131 (0,5\%) óbitos fetais e $172(0,7 \%)$ óbitos neonatais ${ }^{14}$

Mães com Sífilis Gestacional, geralmente, são orientadas quanto à possibilidade de seus filhos desenvolverem sequelas caso nasçam com o diagnóstico da doença ${ }^{3}$. Nesse sentido, destaca-se que a assistência pré-natal de qualidade com captação precoce da gestante, ações de promoção da saúde, orientação sexual e reprodutiva, realização do protocolo dos exames preconizados durante o período gestacional são essenciais para a prevenção de danos ao RN ${ }^{15}$.

\section{A reincidência da $\mathrm{SC}$ em mais de uma gestação}

A puérpera F3 verbalizou que o fato de já ter vivenciado a SC em sua filha mais velha a deixou mais preparada para o enfrentamento do mesmo diagnóstico no RN.

[...] o choque foi tudo na primeira. [...] quando a outra filha nasceu eu estava mais vulnerável. Dele, eu já vim para o hospital sabendo que [...] ele ia ficar os 10 dias. [...] Eu já estava preparada [...]. (F3)

Estudo realizado no Rio de Janeiro mostrou que a ocorrência de gestação prévia com sífilis não eliminou o risco da doença em futuras gestações ${ }^{16}$. Dados semelhantes revelam a reincidência de SC no segundo filho ${ }^{13,17}$. Outra pesquisa expõe que $6.957(41,1 \%)$ das gestantes obtiveram o registro de duas sorologias no seu cartão de pré-natal ${ }^{14}$. 
A reincidência da SC para outras gestações é dada a falhas no que diz respeito a ações educativas de controle e prevenção de novos casos. Estudo apontou desinformação dessas acerca da transmissão vertical, revelando, ainda, a falta de orientações por parte dos profissionais, comprometendo a forma como elas entendem, enfrentam e dão a devida importância ao seguimento do seu tratamento e dos $\mathrm{RN}^{3}$. Apesar de baixa prevalência de reincidência de SC na segunda gestação, essa ainda é presente no estudo, revelando-se falhas nos métodos de prevenção/controle da doença, contribuindo para o aumento de agravos à saúde materno-infantil.

\section{0 medo do estigma que envolve a doença}

Verificou-se a preocupação das mães com o fato das pessoas saberem o diagnóstico da criança. Destacou-se que se sentem estigmatizadas pelos profissionais da saúde que as assistem. A maior parte das mães optou por esconder o diagnóstico dos familiares menos próximos, alegando que a criança estava internada por outro motivo clínico.

Para mim foi pior saber que os outros sabiam. Os familiares não sabem, eu não contei. [...] Então, a gente diz que é por qualquer coisa [...] Mas é constrangedor, porque vão dizer que sou promíscua [...] Principalmente, o julgamento das pessoas que trabalham com isso e não devia ser assim. Eu me senti muito mal [...]. (F14)

Eu não falei porque é sifilis, o que vai vir na mente deles: - Que eu sou podre, sabe? (risos). - Então, eu não falei. (F8)

O medo do estigma da doença pode estar associado a fatores culturais, visto que por muito tempo as Infeções Sexualmente Transmissíveis (IST) predominavam em profissionais do sexo, usuários de drogas e homossexuais. Ainda hoje se verificam pré-julgamentos e associação dessas infecções com promiscuidade e comportamentos de risco. Estudo apontou que a sífilis, na visão dos enfermeiros, tem como fator de risco a baixa condição socioeconômica das gestantes, principalmente das que vivem em promiscuidade sexual, múltiplos parceiros, usuárias de drogas e baixa escolaridade, reforçando o estigma da doença ${ }^{5}$. Comprovou-se que a sífilis ainda encontra-se cercada de desinformação, preconceitos, discriminação e exclusão social ${ }^{18}$.

A confirmação do diagnóstico de doenças como Vírus da Imunodeficiência Humana/Síndrome da Imunodeficiência Adquirida (HIV/AIDS) e Sífilis acarreta importantes alterações biológicas e psicossociais, em razão do estigma associado às IST $^{19}$. Pesquisa apontou a decisão de duas mães em omitirem o verdadeiro diagnóstico do filho, devido ao estigma da doença ${ }^{17}$. Receio de julgamento, rejeição familiar e raiva do companheiro também foram apresentados em estudo frente ao diagnóstico de IST/AIDS por mulheres reclusas. Destacaram o aconselhamento feito pelos profissionais da Enfermagem como importante meio de suporte emocional frente à IST ${ }^{20}$. Profissionais eticamente comprometidos devem defender a dignidade, especialmente de mulheres vulneráveis frente ao estigma vivenciado ${ }^{21}$.

\section{A busca de informações acerca da SC}

As mães buscaram informações sobre a SC na internet. Receberam orientações de médicos e enfermeiros e, também, receberam folders sobre a doença nas Unidades Básicas de Saúde durante o pré-natal.

Foi na internet. Quem me passou informação foi a minha médica do pré-natal. Ela que me falou assim por cima. [...] depois eu puxei na internet e vi. (F12)

[...] eles dão um folhetinho que diz o que pode acontecer com o bebê [...]. Também me informei na internet. Na maternidade os médicos e enfermeiros me avisaram que ele ia ter que ficar aqui dez dias [...]. (F8)

A entrevistada F11 graduada em Enfermagem revelou que mesmo apropriada de conhecimentos não deixou de buscar informações na internet ao receber o diagnóstico de Sífilis na gestação. Realizava orientação de adolescentes a respeito da Educação Sexual nas escolas e Unidades Básicas de Saúde.

A gente recebe informações no curso de graduação. Eu fazia Educação Sexual nas escolas e a gente tinha que levar essas informações. Eu fazia educação para a saúde nas salas de espera das Unidades Básicas. Mas quando recebi o diagnóstico, procurei na internet também. (F11)

Outro aspecto ressaltado foi que mãe F15 não teve coragem de pesquisar sobre a doença e só procurou apropriarse do assunto após o nascimento da criança.

\section{Eu não tive coragem, eu só procurei saber agora. (F15)}

Oferecer aos pais das crianças orientação acerca da SC representa uma importante estratégia de enfrentamento da doença ${ }^{5}$. Estudo apontou que RN com diagnóstico de SC foram internados no hospital em proporção muito mais elevada e tiveram entres duas a três vezes mais desfechos negativos do que os nascidos de mães não infectadas pela sífilis $^{12}$. Enfatiza-se que o enfermeiro deve ser um articulador de processos de trabalho e de responsabilização pela organização das ações de educação em serviço ${ }^{22}$. 0 enfermeiro apresenta uma atuação importante como educador ${ }^{5}$. No entanto, dados revelam que o despreparo técnico dos profissionais da atenção primária pode acarretar ineficiência e baixo desempenho das ações de controle da sífilis ${ }^{19}$. Assim, torna-se necessária a educação permanente e continuada desse profissional, auxiliando na propagação de informações por fontes seguras. 
É importante que a prática do aconselhamento pelos profissionais de saúde durante o pré-natal amplie-se para além de orientações sobre IST e solicitação de exames, mas também esteja vinculado a pacientes soropositivos, à oferta e adesão ao tratamento, assim como à conscientização do parceiro (a) sexual e redução de danos. Deve-se observar a autonomia dos sujeitos, o diálogo sobre as suas práticas de risco, a escolha de métodos de prevenção e o esclarecimento de dúvidas sobre o tratamento e enfrentamento do estigma ${ }^{23}$.

\section{CONCLUSÃO}

As repercussões das mães foram manifestadas por sentimentos de culpa, desespero, tristeza e horror ao receberem o diagnóstico de sífilis congênita no seu filho. Verificou-se que apesar da baixa prevalência, há a reincidência da doença na segunda gestação. Ao enfrentar a SC no RN, as puérperas referiram medo do estigma social e buscaram informações acerca da doença na internet, como também com médicos e enfermeiros.

Concluiu-se que há desinformação das mães quanto à infecção por sífilis, principalmente sobre como evitar a transmissão vertical e a reinfecção durante o tratamento. Existem lacunas na triagem da sífilis gestacional que precisam ser preenchidas, realizando testagem precoce para IST, evitando diagnósticos tardios e contaminação fetal. As políticas públicas não estão sendo efetivas quanto a coibir a contaminação pela SC, carecendo novas estratégias para o combate da infecção nas redes de atenção à saúde, a fim de transmitir informações, promovendo a saúde do binômio mãe-bebê.

Os enfermeiros não estão aproveitando o pré-natal e o período de internação do RN com SC para realizar a prática educativa junto às mães. A educação em saúde revela-se como ferramenta fundamental para difundir conhecimentos. O enfermeiro deve acompanhar o fornecimento do diagnóstico da mãe e do RN, orientando sobre a profilaxia, formas de contágio, sinais e sintomas e tratamento da doença. Deve enfatizar a importância da realização das testagens para IST durante o pré-natal, do tratamento do parceiro, assim como o uso de preservativos durante as relações sexuais. Frente à SC é importante informar os riscos de sua transmissão ao RN, assim como os cuidados necessários que devem ser dispensados a este, enfocando, também, a necessidade do prolongamento da internação para tratamento, os procedimentos e exames que serão realizados para o melhor enfrentamento da doença, por meio de um processo educativo efetivo. Destaca-se a importância do desempenho ético e da educação permanente dos profissionais de saúde, como medidas para auxiliar a garantia da assistência integral e humana durante o enfrentamento da puérpera ao diagnóstico de SC na criança, gerando transformações nos estigmas apresentados.

São imprescindíveis novos estudos para conhecer as repercussões da SC em longo prazo. Teve-se como limitações a negativa de 10 mães selecionadas para participar da pesquisa, como também ter sido realizada em um único contexto não possibilitando a generalização dos achados.

\section{REFERÊNCIAS}

1. Ministério da Saúde (Br). Secretaria de Vigilância em Saúde. Departamento de DST, Aids e Hepatites Virais. Protocolo clínico e diretrizes terapêuticas para atenção integral às pessoas com infecções sexualmente transmissíveis. Relatório de Recomendação. [Online] Brasília (DF): Ministério da Saúde; 2015 [cited 2018 Aug 15]. Available from: http://bvsms.saude.gov.br/bvs/publicacoes/protocolo_clinico_diretrizes_terapeutica_atencao_integral_pessoas_infeccoes_sex ualmente_transmissiveis.pdf.

2. World Health Organization (Who). Sexually Transmitted Infections (STIS). The importance of a renewed commitment to STI prevention and control in achieving global sexual and reproductive health [Online]. 2013. [cited 2018 Sep 20]. Available from http://www.who.int/reproductivehealth/publications/rtis/rhr13_02/en/index.html.

3. Lima VC, Mororó RM, Feijão DM, Frota MVV, Martins MA, Ribeiro SM, et al. Mother's perception of congenital syphilis in her fetus. Revista de Saúde Pública do Paraná [Online]. 2016 [cited 2018 Aug 15]; 17(2):118-25. DOI: http://dx.doi.org/10.22421/1517-7130.2016v17n2p118.

4. Lopes ACMU, Araújo MAL, Vasconcelos LDPG, Uchoa FSV, Rocha HP, Santos JR. Implementation of fast tests for syphilis and HIV in prenatal care in Fortaleza, Ceará. Rev. bras. enferm. [Online]. 2016 [cited 2018 Jul 30]; 69(1):54-8. DOI: http://dx.doi.org/10.1590/0034-7167.2016690108i.

5. Mororó MR, Lima VC, Frota MVV, Linhares MSC, Ribeiro SM, Martins MA. The perception of nurses health strategy family about the following of congenital syphilis. Rev.Saúde.Com. [Online]. 2015 [cited 2018 Jul 30]; 11(3):291-302. Available from: http://www.uesb.br/revista/rsc/v11/v11N3a06.pdf.

6. Figueiredo MSN, Cavalcante EGR, Oliveira CJ, Monteiro MFV, Quirino GS, Oliveira DR. Perception of nurses on the adhesion of partners of pregnant women with syphilis to the treatment. Rev. Rene. [Online]. 2015 [cited 2018 Aug 05]; 16(3): 345-54. DOI: http://dx.doi.org/10.15253/2175-6783.2015000300007.

7. Cavalcante PAM, Pereira RBL, Castro JGD. Syphilis in pregnancy and congenital syphilis in Palmas, Tocantins State, Brazil, 20072014. Epidemiol. Serv. Saúde [Online]. 2017 [cited 2018 Sep 10]; 26(2):255-64. DOI: http://dx.doi.org/10.5123/s167949742017000200003. 
8. Gomes GC, Erdmann AL, Oliveira PK, Xavier DM, Santos SSC, Farias DHR. The family living the time during the hospitalization of the child: contributions for nursing. Esc. Anna Nery Rev. Enferm. [Online]. 2014 [cited 2018 Aug 20];18(2):234-40. DOI: http://dx.doi.org/10.5935/1414-8145.20140034.

9. Azevêdo AVS, Lanconi Júnior AC, Crepaldi MA. Nursing team, family and hospitalized child interaction: an integrative review. Ciênc. saúde coletiva [Online]. 2017 [cited 2018 Oct 02]; 22(11): 3653-66. DOI: http://dx.doi.org/10.1590/1413812320172211.26362015.

10. Workowski KA, Bolan GA. Center for Disease Control and Prevention. Morbidity and Mortality Weekly Report. Sexually Transmitted Diseases Treatment Guidelines, 2015. Recommendations and Reports. [Online]. 2015 [cited 2018 Jul 23]; 64(3):459. Available from: https://www.cdc.gov/std/tg2015/tg-2015-print.pdf.

11. Bardin L. Análise de conteúdo. São Paulo: Edições 70; 2011.

12. Guimarães MSF, Santos IMM, Silva LJ, Christoffel MM, Silva LR. Parenthood of parents of newborns hospitalized due to congenital syphilis in the light of the transition theory. Texto \& contexto enferm. [Online]. 2018 [cited 2018 Aug 23]; 27(4):e1190017. DOI: http://dx.doi.org/10.1590/0104-07072018001190017.

13. Lafetá KRG, Martelli Júnior H, Silveira MS, Paranaíba LMR. Maternal and congenital syphilis, underreported and difficult to control. Rev. bras. epidemiol. [Online]. 2016 [cited 2018 Aug 23]; 19(1):63-74. DOI: http://dx.doi.org/10.1590/19805497201600010006.

14. Domingues RMM, Leal MC. Incidence of congenital syphilis and factors associated with vertical transmission: data from the Birth in Brazil study. Cad. Saúde Pública [Online]. 2016 [cited 2018 Jul 6]; 32(6):e00082415. DOI: http://dx.doi.org/10.1590/0102-311X00082415.

15. Padovani C, Oliveira RR, Pelloso SM. Syphilis in during pregnancy: association of maternal and perinatal characteristics in a region of southern Brazil. Rev. latinoam. enferm. [Online]. 2018 [cited 2018 Sep. 14]; 26:e3019. DOI: http://dx.doi.org/10.1590/1518-8345.2305.3019.

16. Cerqueira LR, Monteiro DLM, Traquette SR, Rodrigues NCP, Trajano AJB, Souza FM, et al. The magnitude of syphilis: from prevalence to vertical transmission. Rev. Inst. Med. Trop. São Paulo. [Online]. 2017 [cited 2018 Oct. 01]; 59:e78. DOI: http://dx.doi.org/10.1590/s1678-9946201759078.

17. Nonato SM, Melo APS, Guimarães MDC. Syphilis in pregnancy and factors associated with congenital syphilis in Belo HorizonteMG, Brazil, 2010-2013. Epidemiol. Serv. Saúde [Online]. 2015 [cited 2018 Jul 02]; 24(4). Available from: http://www.scielo.br/scielo.php?pid=S2237-96222015000400681\&script=sci_abstract.

18. Ministério da Saúde (Br). Secretaria de Vigilância em Saúde. Boletim Epidemiológico- Sífilis. [Online] Brasília (DF): Ministério da Saúde; 2017 [cited 2018 Sep 22]. Available from: http://www.aids.gov.br/pt-br/pub/2017/boletim-epidemiologico-de-sifilis2017.

19. Silva AP, Corrêa CM, Barbosa JAG, Borges CM, Souza MCMR. Counseling on HIV / AIDS and syphilis to pregnant women in primary care. Rev. enferm. UFPE [Online]. 2018 [cited 2018 Oct 01]; 12(7):1962-9. DOI: https://doi.org/10.5205/1981-8963v12i7a236251p1962-1969-2018.

20. Fernandes MA, Bezerra MM, Moura FMJSP, Alencar NES, Lima FFF, Castro AED. Sexually transmitted infections and the experiences of women in situations of imprisonment. Rev. enferm. UERJ [Online]. 2016 [cited 2018 Sep 20]; 24(6):e27774. DOI: http://dx.doi.org/10.12957/reuerj.2016.27774.

21. Cook RJ, Dickens BM. Reducing stigma in reproductive health. Int. J. Gynaecol. Obstet. [Online]. 2014 [cited 2018 Sep 15]; 125(1):89-92. DOI: http://dx.doi.org/10.1016/j.ijgo.2014.01.002.

22. Fagundes NC, Rangel AGC, Carneiro TM, Castro LMC, Gomes BS. Continuing professional development in health for working nurses. Rev. enferm. UERJ [Online]. 2016 [cited 2018 Oct 01]; 24(1). DOI: http://dx.doi.org/10.12957/reuerj.2016.11349.

23. Ministério da Saúde (Br). Secretaria de Vigilância em Saúde. Departamento de Vigilância, Prevenção e Controle das Infecções Sexualmente Transmissíveis, do HIV/Aids e das Hepatites Virais. Diretrizes para organização e Funcionamento dos CTA no âmbito da Prevenção Combinada e nas Redes de Atenção à Saúde [Online]. Brasilia (DF): Ministério da Saúde; 2017 [cited 2018 Jul 5]. Available from: http://www.aids.gov.br/ptbr/gestores/diretrizes-para-organizacao-efuncionamento-dos-cta-no-ambitodaprevencao-combinada. 